\title{
VIRTUAL HYDRAULICS IN HARDWARE-IN-THE-LOOP SIMULATION OF MECHATRONICS MACHINES
}

\author{
T. Eskola, H. Handroos, A. Rouvinen \\ Department of Mechanical Engineering \\ Lappeenranta University of Technology \\ PO Box 20, FIN-53851, Lappeenranta, Finland \\ (E-mail: tero.eskola@lut.fi)
}

\begin{abstract}
When hydraulically driven machine is constructed it is often hard to study the effects of alternative hydraulic components and systems on its behavior because it is time-consuming to install and replace the components in the real construction. An effective and timesaving method is presented. The method is based on a real-time hydraulics simulation model which is simulated while running the real machine construction. The main idea of this method is to get the real hydraulics, connected with the mechanism, to behave like the virtual hydraulics does. When managed, the variations can easily be made in the simulation model and their effects then be seen from the behavior of real construction. Also, properties of mechanics like flexibility, gaps and friction quite often difficult to model are automatically included.
\end{abstract}

\section{KEY WORDS}

Virtual hydraulics, Semi-empirical modeling, Hardware-in-the-loop simulation

\section{NOMENCLATURE}

\author{
$B_{e}:$ Effective bulk modulus \\ $C$ : Semi-empirical parameter \\ $k$ : Empirical constant \\ $K$ : Semi-empirical parameter \\ $p$ : Pressure \\ $P$ : Power \\ $Q$ : Oil flow \\ $U$ : Control signal \\ $V$ : Volume of chamber
}

\author{
$\eta:$ Efficiency \\ $\tau$ : Time-constant
}

\section{INTRODUCTION}

By using a simulation model of entire construction it is easy to study the effects of component variations. If however, the construction is complex, it is often timeconsuming to built a simulation model of the complete machine system. Real-time and hardware-in-the-loop simulation have recently been applied into hydraulic driven machine systems [1], [2] and [3]. Generally, the 
most common applications of these technologies are in the field of testing practical digital control systems. They are rarely used in R\&D of hydraulic or mechanical systems.

The main idea of developed method is to calculate the inputs into real hydraulics by means of a real-time simulated virtual hydraulics such that it behaves and drives the mechanical system as the virtual hydraulics would do. Thus, the variations can be made in the simulation model and their effects then be studied in the real construction.

In order to simulate hydraulics as a part of larger machine system variable values must be transmitted between the real and virtual part. The interface between the simulation model and real construction must be capable of two-way data delivery. The input signals to the simulation model are the real pressures and the output signals are the reference flows which are transformed into real flows by means of highbandwidth servovalves. The circulation of information signals of the used system is presented in figure 1 .

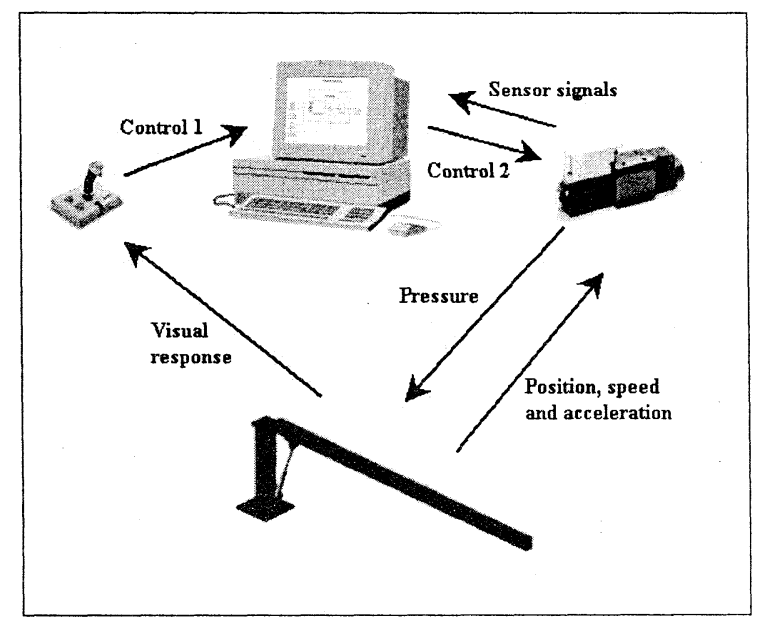

Figure 1 The circulation of information signals

\section{REAL-TIME SIMULATION OF HYDRAULICS}

Because there are only a few commercial tools for realtime simulation of hydraulics, the simulation models used in this work have been created by authors. Models were built by employing semi-empirical approach. The benefit of semi-empirical modeling method is that the models are faster to use because almost every parameters can be seen from the component manufacturer's catalogue and therefore without dismantling the component. The most important equations used in this study are presented below [4], [5] and [6].
The flow through the pressure relief valve is:

$$
Q=K \sqrt{p_{1}}
$$

Where $K$ can be solved from:

$\frac{\ddot{K}+2 C_{3} C_{4} \dot{K}}{C_{3}^{2}}+\left(C_{1}+C_{2} p_{1}\right) K=p_{1}-p_{r e f}$

The flow through one orifice of the directional valve is:

$$
Q=U \cdot C_{V} \cdot \sqrt{\Delta p}
$$

Where $U$ can be solved from:

$$
\dot{U} \doteq \frac{U_{1}-U}{\tau}
$$

Where $U_{1}$ is the reference signal given by user and $\tau$ can be solved from:

$$
\tau=\frac{1}{2 \pi f_{-45^{\circ}}}
$$

Where $f_{-45^{\circ}}$ is the $-45^{\circ}$ bandwidth of the valve.

The pressure in the chamber is:

$\dot{p}=\frac{B_{e}}{V_{\text {tot }}}\left(\sum Q_{I N}-\sum Q_{\text {OUT }}-\frac{d V}{d t}\right)$

The pump is a variable displacement pump equipped with constant pressure controller. A simplified dynamic model taking into account the controller dynamics and flow as power limits can be expressed as follows:

$$
Q_{p}=\min \left\{\begin{array}{c}
\frac{P_{\max } \cdot \eta_{\text {tot }}}{p_{p}} \\
Q_{\max } \\
\int_{0}^{T} \frac{k_{p}\left(p_{r e f}-p_{p}\right)-Q_{p}}{\tau_{t}} d t
\end{array}\right.
$$




\section{MAKING REAL HYDRAULICS MIMIC VIRTUAL}

The structure of the used HIL-simulation is presented in figure 2. Unlike normal situation the user controls virtual hydraulics which produce virtual flow $Q_{1}$. The behavior of virtual hydraulics depends on the measured pressures and the solved virtual flow is used as a reference flow according to which a control signal $U_{2}$ is created for real hydraulics so that the real flow $Q_{2}$ becomes as close to $Q_{1}$ as possible.

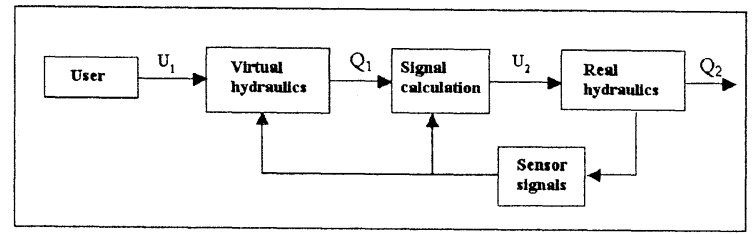

Figure 2 Structure of used HIL-simulation

The new command signal $U_{2}$ for the real servovalve can be generated by using a flow feedback system with a flow transducer and a PID-controller. The method does not need the knowledge of the used servovalve but it requires fast information about the crossflow.

Another method to define the command signal $U_{2}$ from the virtual flow is to calculate it by employing the inverse of the semi-empirical directional valve flow model, (8). Equation (8) takes into account the effects of valve leakage and dynamics.

$$
U_{2}=\frac{Q_{1}+Q_{\text {leak }}}{C_{V R} \sqrt{\Delta p}}+\frac{\frac{d}{d t}\left(\frac{Q_{1}+Q_{\text {leak }}}{C_{V R} \sqrt{\Delta p}}\right)}{2 \pi f_{-45_{R}^{o}}}
$$

\section{EXPERIMENTAL WORK}

The natural approach in modeling hydraulics is to use separate component models which are connected by means of continuity equations to build up the circuits. In this work the modeled circuit, presented in figure 3, is simple and widely used.

In the experimental setup user gives the command signal $U_{1}$ to the virtual hydraulics using a joystick. The command signal for the real servo valve is generated by using a flow feedback system with a flow transducer and a PID-controller. The used reference flow is the flow $Q_{1}$, presented in figure 3 . The response time of used piston-type flow transducer was less than $1 \mathrm{~ms}$. The second method based on equation 8 is proposed as an alternative method.

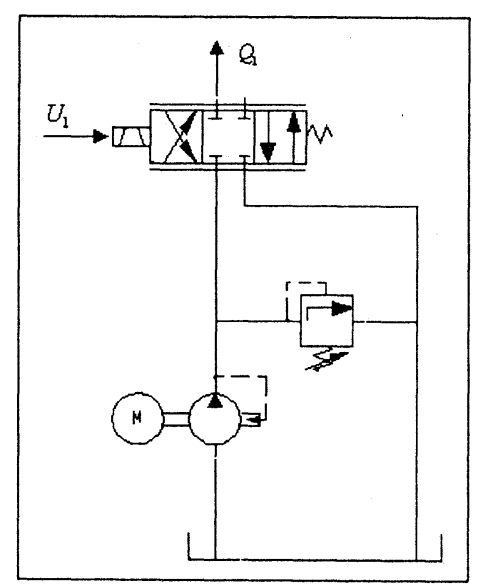

Figure 3 Modeled hydraulic circuit

The defined method can be applied in systems where the reference flows solved by virtual hydraulics can be transformed into real flows. In this work the system, presented in figure 4 , was consisted of simple crane with hydraulic cylinder and servovalve.

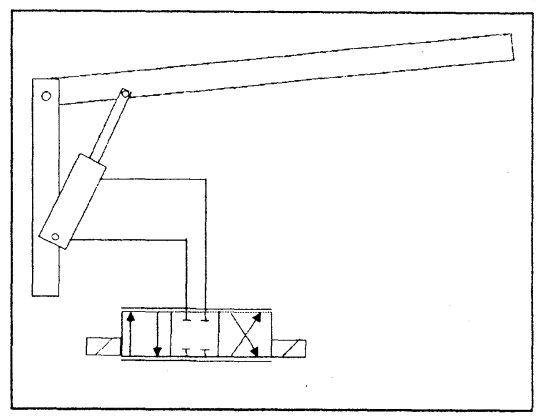

Figure 4 Experimental setup

The used experimental environment is presented in figure 5 .

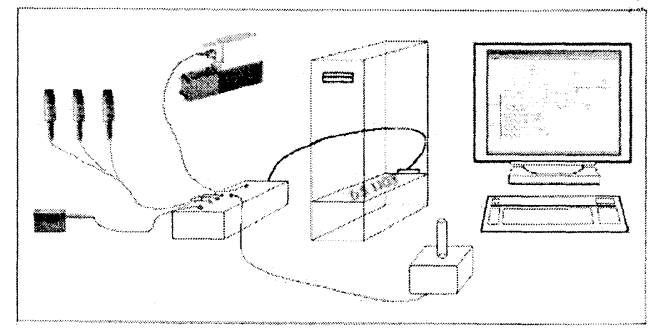

Figure 5 Scheme of the used hardware 


\section{RESULTS}

A new R\&D tool for the hydraulically driven machine systems designers was proposed in this work. The proposed method uses a partly virtual prototype of machines. The key idea is to make the real hydraulics mimic simulator output flows. Also two methods for computing the new control signals for the real hydraulics were defined and the result flows are compared with the reference flows in figures 6 and 7.

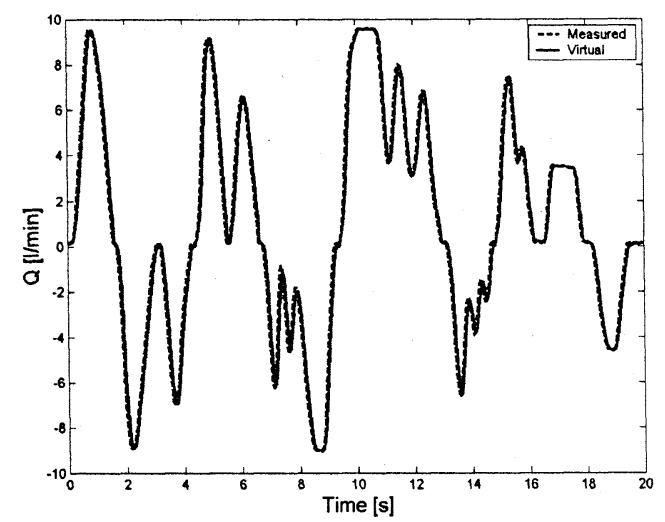

Figure 6 Measured flow reached by flow feedback system with PID-control compared with virtual flow

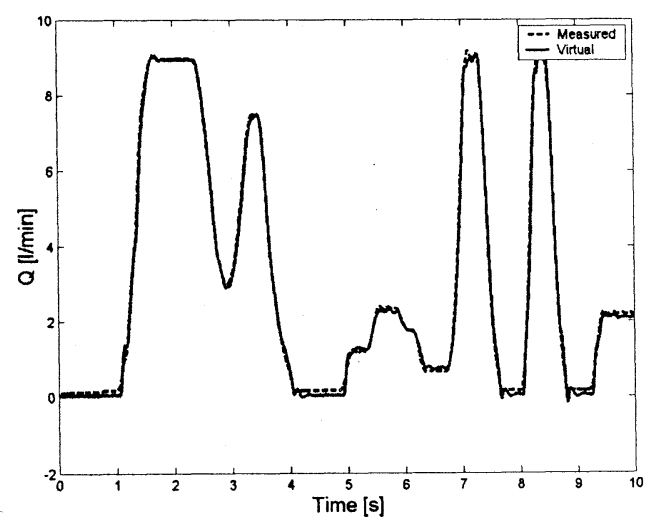

Figure 7 Measured flow reached by employing the semi-empirical flow model compared with virtual flow

\section{CONCLUSION}

An effective and time saving method for designing and developing of hydraulically driven machine was presented. The method is based on a real-time simulation model of hydraulics which is simulated while running the real machine construction. By using the proposed method the variations can be made in the virtual hydraulics and their effects then be studied from the behavior of the real construction. By using existing construction it requires less modeling work and properties of mechanics like flexibility, gaps and friction quite often difficult to model are automatically included. Also two methods for calculating the new control signals for real servovalve were defined. Simple test systems were introduced and promising results achieved.

\section{REFERENCES}

1. Helduser, S., Stuewing, M., Liebig, S., Dronka, S. Development of Electrohydraulic Actuators Using Simulation and Hardware-in-the-loop Technology, PTMC, 2001

2. Hämäläinen, M., Liu, Y., Handroos, H., Rouvinen, A. Real-time Simulation of a Hydraulically Driven Log Crane by Utilizing Commercically Available Software, PTMC, 2001

3. Linjama, M., Virvalo, T. Hardware-in-the-loop simulation Applied on Distributed Control of Hydraulic Cylinder Drives, 2nd International Fluid Power Conference, 2000

4. Handroos, H., Vilenius, M. The Utilization of Experimental Data in Modelling Hydraulic Single Stage Pressure Control Valves. ASME Journal of Dynamic Systems, Measurements and Control, Vol. 112, Number 3, 1990

5. Handroos, H., Mikkola, A., Liukkula, M. Virtual Prototyping of hydraulic Driven Mechatronic Machines. $4^{\text {th }}$ JHPS International Symposium on Fluid Power, 1999.

6. Mikkola, A. Handroos, H. Modelling and Simulation of a Flexible Hydraulic Driven Log Crane, $9^{\text {th }}$ Bath International Fluid Power Workshop, 1996. 